\title{
Lithium chloride attenuates the abnormal osteogenic/adipogenic differentiation of bone marrow-derived mesenchymal stem cells obtained from rats with steroid-related osteonecrosis by activating the $\beta$-catenin pathway
}

\author{
ZEFENG YU* , LIHONG FAN*, JIA LI, ZHAOGANG GE, XIAOQIAN DANG and KUNZHENG WANG \\ Department of Orthopedics, The Second Affiliated Hospital of Xi'an Jiaotong University,
} Xi'an, Shaanxi 710004, P.R. China

Received July 6, 2015; Accepted August 27, 2015

DOI: $10.3892 /$ ijmm.2015.2340

\begin{abstract}
Steroid-related osteonecrosis of the femoral head $(\mathrm{ONFH})$ may be a disease that results from the abnormal osteogenic/adipogenic differentiation of bone marrow-derived mesenchymal stem cells (BMMSCs). In the present study, we examined the possible use of lithium in an aim to reverse the abnormal osteogenic/adipogenic differentiation of BMMSCs isolated from rats with steroid-related ONFH (termed ONFHBMMSCs). BMMSCs obtained from steroid-related ONFH rat femurs were cultured with or without lithium chloride $(\mathrm{LiCl})$. BMMSCs obtained from normal rat femurs were cultured as controls. LiCl significantly increased the expression of osteocalcin and Runx 2 in the ONFH-BMMSCs during osteogenic induction. The mineralization of ONFH-BMMSCs following osteogenic induction was also enhanced. Furthermore, $\mathrm{LiCl}$ exerted anti-adipogenic effects on the ONFH-BMMSCs by inhibiting the expression of peroxisome proliferator-activated receptor $\gamma($ PPAR $\gamma$ ) and fatty acid binding protein 4 (Fabp4) during adipogenic induction, and decreasing lipid droplet
\end{abstract}

Correspondence to: Dr Kunzheng Wang, Department of Orthopedics, The Second Affiliated Hospital of Xi'an Jiaotong University, No. 157 Xiwu Road, Xi'an, Shaanxi, 710004, P.R. China

E-mail: wkzh1955@mail.xjtu.edu.cn

"Contributed equally

Abbreviations: ONFH, osteonecrosis of the femoral head; BMMSCs, bone marrow-derived mesenchymal stem cells; GSK-3 $\beta$, glycogen synthase kinase $3 \beta$; MSC, mesenchymal stem cell; MTT, methyl thiazolyl tetrazolium; OCN, osteocalcin; IBMX, 3-isobutyl-1-methylxanthine; DMEM, Dulbecco's modified Eagle's medium; FBS, fetal bovine serum; SDS-PAGE, sodium dodecyl sulphate-polyacrylamide gel electrophoresis; QUE, quercetin; BGLAP, bone gamma-carboxyglutamic acid-containing protein

Key words: lithium choride, abnormal osteogenic/adipogenic differentiation, bone marrow-derived mesenchymal stem cells, steroid-related osteonecrosis, $\beta$-catenin pathway formation at the end of adipogenic induction. These effects of $\mathrm{LiCl}$ on the ONFH-BMMSCs were associated with an increased expression of $\beta$-catenin and a decreased expression of phosphorylated GSK-3 $\beta$ at Tyr-216, and these effects were abolished by treatment with quercetin, an antagonist of the $\beta$-catenin pathway. The normal osteogenic/adipogenic activity of BMMSCs may be impaired in steroid-related ONFH. However, as demonstrated by our findings, $\mathrm{LiCl}$ reduces abnormal adipogenic activity and simultaneously increases the osteogenic differentiation of ONFH-BMMSCs by activating the $\beta$-catenin pathway.

\section{Introduction}

Recently, steroid-related osteonecrosis of the femoral head $(\mathrm{ONFH})$ has been reported to occur in patients with collagen vascular diseases following high-dose steroid treatment (1). Several treatment strategies for ONFH have been implented, including drug therapy and surgery. However, these methods have barely been effective in treating the disease. As steroid-related ONFH usually occurs in young and middle-aged patients and is associated with a poor prognosis (2), it is important to examine the pathophysiology of steroid-related ONFH and develop a preventive strategy.

Bone marrow-derived mesenchymal stem cells (BMMSCs) are multipotent cells that can differentiate into adipocytes or osteocytes (3). Changes in the functional characteristics of the differentiation pathway of BMMSCs may contribute to the pathogenesis of ONFH (4). Furthermore, interactions involving the differentiation pathway of BMMSCs that promote adipogenesis and decrease osteogenesis, are considered to be the major factors leading to steroid-related ONFH (5). Adipogenesis and osteogenesis share an inverse relationship in the bone marrow during the differentiation of BMMSCs (6). In ONFH, BMMSCs may differentiate preferentially into adipocytes rather than osteoblasts (7). Thus, the enhancement of osteogenesis coupled with a reduction in adipogenesis may provide a potential therapeutic strategy for the treatment of ONFH.

Wnt/ $\beta$-catenin signaling functions as a molecular switch that determines the balance between osteogenesis and adipo- 
genesis (8). Moreover, Wnt/ $\beta$-catenin signaling is reportedly activated in osteoblast-committed MSCs, further indicating that it controls the balance between the osteogenesis and adipogenesis of MSCs (9). $\beta$-catenin is phosphorylated by a complex composed of adenomatous polyposis coli protein, glycogen synthase kinase $3 \beta$ (GSK-3 $\beta$ ) and axin. Following phosphorylation, $\beta$-catenin is subsequently decomposed $(10,11)$. Lithium, which has been used in the treatment of bipolar disorder for years (12), may be used to inactivate GSK-3 $\beta$, thus resulting in the accumulation of cytoplasmic $\beta$-catenin (13). Galli et al reported that lithium chloride $(\mathrm{LiCl})$ enhanced osteogenesis in osteoblast-like MC3T3-E1 mouse cells (14). In addition, Itoigawa et $a l$ and $\mathrm{Sen}$ et al demonstrated that $\mathrm{LiCl}$ reduces the efficacy of adipogenic induction $(15,16)$. However, $\mathrm{LiCl}$ has rarely been used in the treatment of ONFH, and the detailed mechanisms remain to be elucidated.

The benefits of $\mathrm{LiCl}$ are manifold; based on this fact, we thus hypothesized that $\mathrm{LiCl}$ may attenuate the abnormal osteogenic and adipogenic differentiation of BMMSCs obtained from rats with steroid-related ONFH through the activation of the $\beta$-catenin pathway.

\section{Materials and methods}

Animals. All the experimental procedures adhered to the recommendations of the Experimental Animal Center of Xi'an Jiaotong University, Xi'an, China, and were approved by the Ethics Committee of Xi'an Jiaotong University. A total of 45 male Sprague Dawley rats weighing 190-210 g were obtained from the Experimental Animal Center of Xi'an Jiaotong University. All the animals were housed under specific pathogen-free conditions in a clean, temperature and humidity-controlled environment with unlimited access to food and water and with a 12-h light/dark cycle.

Rat model of ONFH. A total of 30 rats underwent sequential drug administration to establish the ONFH model of ONFH as previously described (17). The remaining 15 rats were used as the normal controls. Briefly, the 30 rats were administered $2 \mathrm{mg} / \mathrm{kg}$ lipopolysaccharide (Sigma, St. Louis, MO, USA) intravenously for 2 days. One day later, $20 \mathrm{mg} / \mathrm{kg}$ of methylprednisolone (Pfizer, Inc., New York, NY, USA) was injected intramuscularly 3 times for $24 \mathrm{~h}$. All the animals were sacrificed (via an intravenous injection of excess phenobarbital sodium) for the isolation of the BMMSCs 4 weeks after the final day of drug administration.

Preparation of rat BMMSCs. The BMMSCs were prepared as previously described (18). Briefly, BMMSCs were obtained from the femoral shafts of the rats (from the rats with ONFH and the normal rats) after the muscles and extraosteal tissues were trimmed. Bone marrow was flushed and centrifuged on a $1.073 \mathrm{~g} / \mathrm{ml}$ Percoll density gradient (Pharmacia, St. Louis, MO, USA). The BMMSCs obtained from the rats with steroid-related ONFH are hereon referred to as ONFHBMMSCs. The cells were washed with PBS (Wuhan Boster Biological Technology, Ltd., Wuhan, China), seeded into $25-\mathrm{cm}^{2}$ cell culture flasks, and cultivated in L-DMEM (Sigma) supplemented with $10 \%$ FBS (Sigma) and $20 \mathrm{mg}$ penicillinstreptomycin $/ \mathrm{ml}$ (Sigma) in a humidified $5 \% \mathrm{CO}_{2}$ atmosphere at $37^{\circ} \mathrm{C}$. The medium was changed every 3 days. When the cells became subconfluent, the cells were released from the culture substratum using trypsin/EDTA (0.25\% trypsin and $0.02 \%$ EDTA) (Sigma). The cell surface molecules, CD44 and CD34, were analyzed on 3 cultures by flow cytometry (FACSCalibur; Becton, Dickinson and Company, Franklin Lakes, NJ, USA).

ONFH-BMMSC viability assay. The ONFH-BMMSCs were plated into 96-well plates at a density of 2,000 cells/well. Following $4 \mathrm{~h}$ of incubation, some of the plates containing the ONFH-BMMSCs were treated with various concentrations of $\mathrm{LiCl}$ (1,5, 10 and $20 \mathrm{mM}$; Sigma). The cells were then incubated at $37^{\circ} \mathrm{C}$ for 5 days. Following incubation, the cells were treated with methyl thiazolyl tetrazolium (MTT; $0.5 \mathrm{mg} / \mathrm{ml}$ ) for $4 \mathrm{~h}$ at $37^{\circ} \mathrm{C}$. Following solubilization with dimethyl sulfoxide, the absorbance was recorded at $570 \mathrm{~nm}$ using a microplate spectrophotometer (SpectraMax; Molecular Devices LLC, Sunnyvale, CA, USA).

Analysis of osteogenic differentiation. The BMMSCs were trypsinized and replated in a 6 -well plate at a concentration of $2 \times 10^{5}$ cells/well. The medium was replaced with osteogenic medium (low-glucose DMEM containing 5\% FBS, $10 \mathrm{nM}$ dexamethasone, $50 \mu \mathrm{M}$ L-ascorbic acid-2-phosphate and $10 \mathrm{mM}$ glycerophosphate; all from Sigma). The medium was added every other day, and the cells were maintained at $37^{\circ} \mathrm{C}$ in a humidified $5 \% \mathrm{CO}_{2}$ atmosphere. The differentiating cells were treated for 7, 14 and 21 days with osteogenic medium alone or medium containing $5 \mathrm{mM} \mathrm{LiCl}$. The rat BMMSCs were divided into the following 3 groups: i) the normal group; ii) the ONFH-BMMSC group; and iii) the $\mathrm{LiCl}+\mathrm{ONFH}-$ BMMSC group. The expression levels of osteocalcin (OCN) and Runx 2 were measured by reverse transcription-quantitative polymerase chain reaction (RT-qPCR).

After 21 days, mineralization was measured using Alizarin Red S (Sigma) staining. Briefly, the BMMSC cultures were fixed in a $4 \%$ paraformaldehyde solution and stained with a $0.5 \%$ Alizarin Red (Sigma) solution. We added $1 \mathrm{ml}$ of $10 \%$ cetylpyridinium chloride (Sigma) for quantitative analysis. The light absorbance of the extracted dye was measured using a spectrophotometer (UV-1800: Shimadzu, Kyoto, Japan) at $570 \mathrm{~nm}$.

Analysis of adipogenic differentiation. The BMMSCs were seeded in 6-well plates at a concentration of $2 \times 10^{5}$ cells/well and cultured in high-glucose DMEM supplemented with $10 \%$ FBS, $1 \%$ penicillin-streptomycin, $10 \mu \mathrm{g} / \mathrm{ml}$ insulin, $1 \mu \mathrm{M}$ dexamethasone and $0.5 \mathrm{mM} 3$-isobutyl-1-methylxanthine (IBMX; all from Sigma) at $37^{\circ} \mathrm{C}$ in a humidified $5 \% \mathrm{CO}_{2}$ atmosphere. The medium was changed every 2 days. The differentiating cells were treated for 3, 7 and 14 days with adipogenic medium alone or medium containing $5 \mathrm{mM} \mathrm{LiCl}$. The expression levels of proliferator-activated receptor $\gamma(\operatorname{PPAR} \gamma)$ and fatty acid binding protein 4 (Fabp4) were measured by RT-qPCR.

After 14 days, the BMMSCs were stained using Oil Red O. Briefly, the cells were fixed with $10 \%$ formalin. The cells were then washed with $60 \%$ isopropyl alcohol and stained with $2 \%$ Oil Red O reagent (Sigma). The staining was quantified by extracting the Oil Red O stain with $100 \%$ isopropyl alcohol, 
after which the absorbance was measured using a spectrophotometer (UV-1800; Shimadzu) at $510 \mathrm{~nm}$.

$R T-q P C R$. Briefly, total cellular RNA was extracted from the cultured cells using TRIzol reagent. Total RNA was then reverse transcribed into cDNA using an RT-PCR mixture (Takara Bio, Otsu, Japan). qPCR was performed in a reaction mixture containing SYBR-Green PCR Master Mix (Roche Diagnostics, Basel, Switzerland). $\beta$-actin was used as an internal control. The following primers were used in the present study: OCN sense, 5'-GAC AAG TCC CAC ACA GCA AC-3' and antisense, 5'-CCG GAG TCT ATT CAC CAC CT-3'; Runx2 sense, 5'-TCA CAA ATC CTC CCC AAG TGG-3' and antisense, 5'-GAA TGC GCC CTA AAT CAC TGA-3'; PPAR $\gamma$ sense, 5'-TCC TCC TGT TGA CCC AGA GCA T-3' and antisense, 5'-AGC TGA TTC CGA AGT TGG TGG-3'; Fabp4 sense, 5'-GGA ATT CGA TGA AAT CAC CCC-3' and antisense, 5'-TGG TCG ACT TTC CAT CCC ACT-3'; and $\beta$-actin sense, 5'-AGT ACC CCA TTG AAC ACG GC-3' and antisense, 5'-TTT TCA CGG TTA GCC TTA GG-3'. The results were analyzed using SDS 2.0 software (Life Technologies, Grand Island, NY, USA).

Western blot analysis. To measure the protein expression of phosphorylaed GSK-3 $\beta$ at Tyr-216 (p-Tyr216 GSK-3 $\beta$ ) and $\beta$-catenin, we conducted western blot analysis. Total proteins were separated on $10 \%$ SDS-PAGE gels and then transferred onto polyvinylidene fluoride blotting membranes. After blocking non-specific binding with 5\% BSA in Tween-Trisbuffered saline, the membranes were incubated with the following primary antibodies: anti-p-Tyr216 GSK-3 $\beta$ (ab75745; 1:500 dilution; Abcam, Cambridge, CA, USA), anti- $\beta$-catenin (ab32572; 1:1,000 dilution; Abcam) and anti- $\beta$-actin (BM0627; 1:200 dilution; Wuhan Boster Biological Technology, Ltd.) antibodies overnight. The membranes were then incubated for $2 \mathrm{~h}$ with secondary antibodies (ZDR-5306, 1:2,000 dilution; Zhongshan Golden Bridge Biotechnology, Co., Ltd., Beijing, China) labeled with horseradish peroxidase. The immunoreactive proteins on the blots were visualized using $\mathrm{ECL}^{\mathrm{TM}}$ western blot detection reagents, and the signals were detected using Image Station 4000R (Kodak, Rochester, NY, USA).

Inhibition of $\beta$-catenin pathway activation. Quercetin (Que; Calbiochem, San Diego, CA, USA), an inhibitor of the $\beta$-catenin pathway $(19,20)$, was used to further investigate the role of $\mathrm{LiCl}$ in the $\beta$-catenin pathway. The ONFH-BMMSCs were treated with $5 \mathrm{mM} \mathrm{LiCl}$ in the presence of Que $(50 \mu \mathrm{M})$ and then underwent osteogenic and adipogenic differentiation. The $\mathrm{LiCl}+\mathrm{ONFH}-$ BMMSC and ONFH-BMMSC groups were used as the controls in this experiment. The mRNA expression levels of Runx2 and PPAR $\gamma$ were measured by RT-qPCR at the end of induction. The expression levels of OCN and Fabp4 were measured by immunofluorescence. Briefly, the BMMSCs were fixed and treated with $50 \mu \mathrm{g} / \mathrm{ml} \mathrm{4',6-diamidino-2-phenylindole} \mathrm{(DAPI)} \mathrm{for} \mathrm{nuclear}$ staining. The primary antibodies were diluted 1:100 [anti-OCN (ab13418) and anti-Fabp4 (ab92501), from Abcam]. The cells were then stained with anti-OCN and anti-Fabp4 antibodies and visualized with a secondary antibody conjugated with tetraethyl rhodamine isothiocyanate (TRITC). The controls were also stained, but without the primary antibodies. Fluorescence images

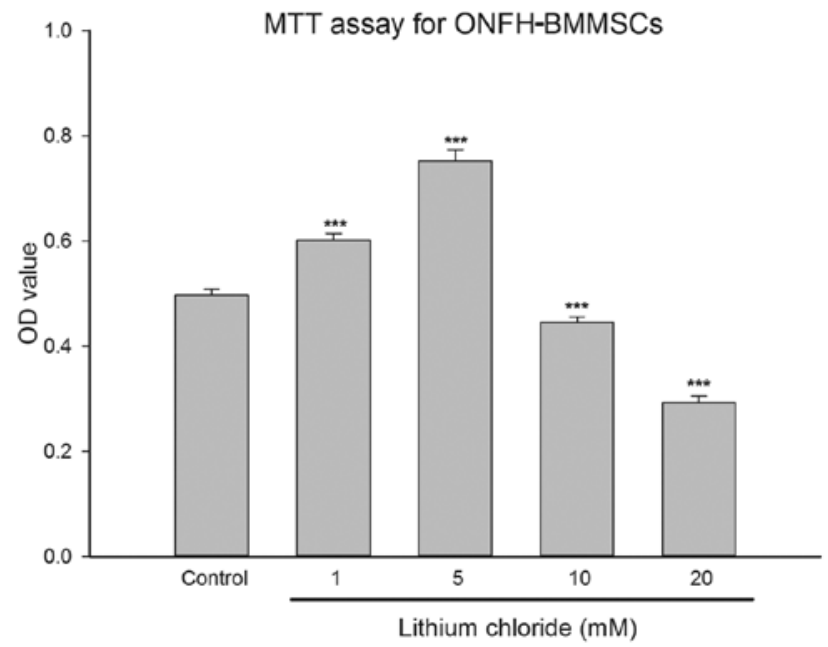

Figure 1. Effects of lithium chloride on the viability of bone marrow-derived mesenchymal stem cells (BMMSCs) isolated from rats with steroid-related osteonecrosis of the femoral head (ONFH-BMMSCs). The ONFH-BMMSCs were treated with various doses of $\mathrm{LiCl}(1,5,10$ and $20 \mathrm{mM})$. Cell viability was analyzed by MTT assay. Of all the treatment groups, the $5 \mathrm{mM}$ concentration of lithium chloride showed the most prominent stimulatory effect on viability. The OD values are shown as the means $\pm \mathrm{SD},{ }^{* * *} \mathrm{P}<0.001$ compared with the controls (normal BMMSCs).

were acquired using a fluorescence microscope (FluoView 500; Olympus, Tokyo, Japan). Data were analyzed using Image-Pro Plus 6.0 (Media Cybernetics, Inc., Rockville, MD, USA).

Statistical analysis. The data are expressed as the means \pm standard deviation (SD). Data analysis was performed using SPSS 13.0 software (SPSS Inc., Chicago, Il, USA). One-way analysis of variance followed by an LSD test was used for comparisons among the experimental groups. P-values $<0.05$ were considered to indicate statistically significant differences.

\section{Results}

Characterization of rat BMMSCs. The BMMSCs were successfully and rapidly expanded into colonies of confluent spindle-shaped cells. The results of flow cytometry for CD34 and CD44 revealed that the cells were negative for CD34 (0.57\%) and positive for CD44 (99.43\%) (data not shown). The cultured cells were thus considered BMMSCs.

Effect of LiCl on the viability of ONFH-BMMSCs. We tested a range of $\mathrm{LiCl}$ concentrations to determine the optimal concentration to treat the ONFH-BMMSCs with. The OD value provided by the MTT assay in the low-dose LiCl-treated groups (1 and $5 \mathrm{mM}$ ) was significantly higher than that of the control group $(\mathrm{P}<0.001$; Fig. 1). Treatment with $\mathrm{LiCl}$ at up to $5 \mathrm{mM}$ increased cell viability in a dose-dependent manner. On the contrary, in the high-dose $\mathrm{LiCl}$-treated groups (10 and $20 \mathrm{mM}), \mathrm{LiCl}$ exerted an inhibitory effect on cell viability $(\mathrm{P}<0.001$; Fig. 1).

LiCl enhances the osteogenesis of ONFH-BMMSCs. The results revealed that the $5 \mathrm{mM} \mathrm{LiCl}$ treatment group and the normal group exhibited a significant increase in the mRNA expression of OCN and Runx 2 in a time-dependent manner compared with the ONFH-BMMSC group ( $\mathrm{P}<0.001$; Fig. 2). Furthermore, we 

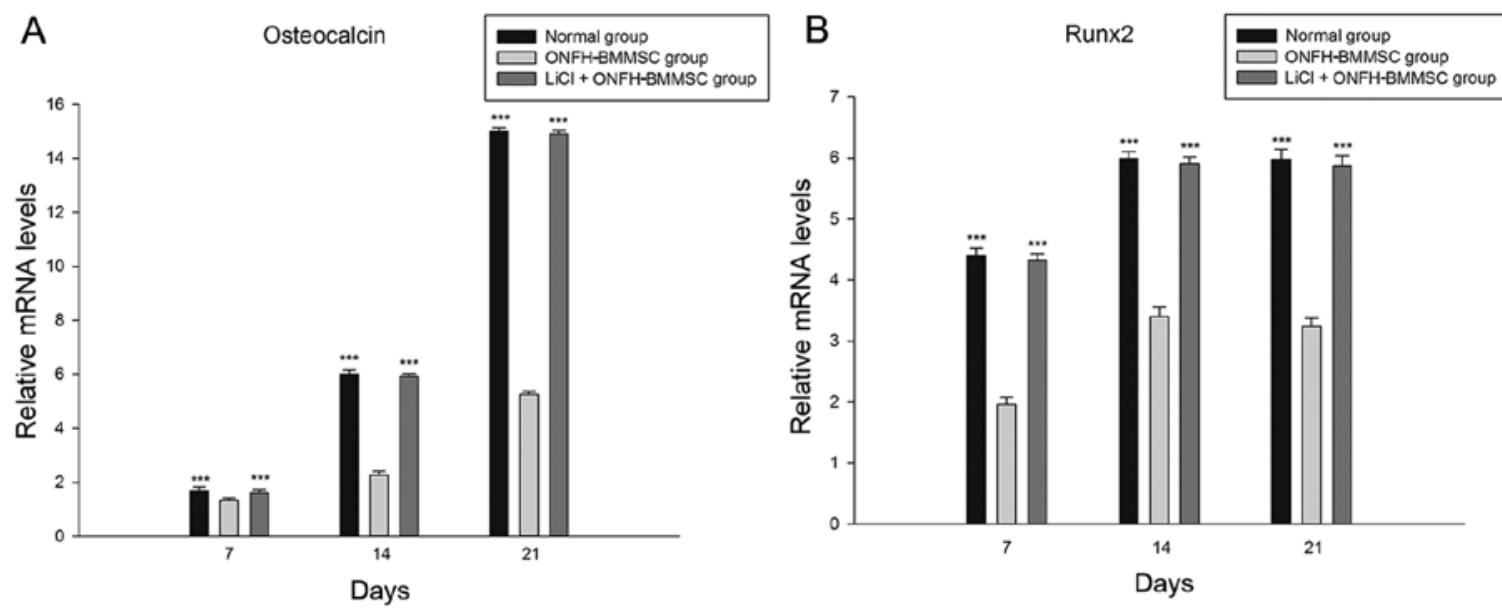

Figure 2. RT-qPCR of osteogenesis-related markers. RT-qPCR was used to determine the osteogenic effects of bone marrow-derived mesenchymal stem cells (BMMSCs) on the mRNA expression of (A) osteocalcin and (B) Runx2 at different time points during osteogenic induction. Gene expression was normalized to $\beta$-actin. The data are shown as the means $\pm \mathrm{SD},{ }^{* * *} \mathrm{P}<0.001$ compared with the BMMSCs isolated from rats with steroid-related osteonecrosis of the femoral head (ONFH-BMMSC) group. LiCl, lithium chloride.
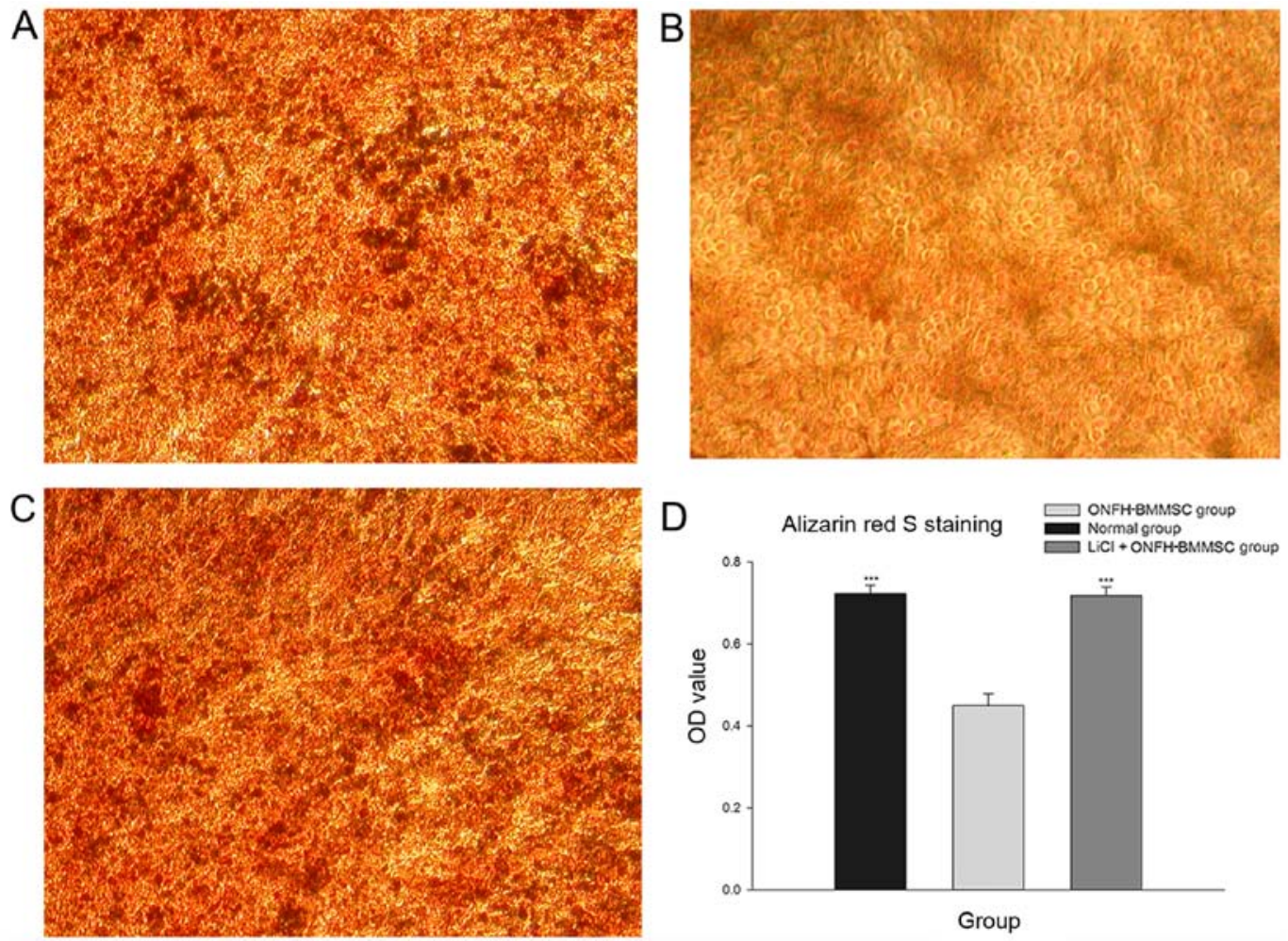

Figure 3. Alizarin Red S staining of rat bone marrow-derived mesenchymal stem cells (BMMSCs) to determine the extent of mineralization. The BMMSCs isolated from rats with steroid-related osteonecrosis of the femoral head (ONFH-BMMSC) group showed lighter Alizarin Red S staining when compared with the normal group or the $\mathrm{LiCl}+$ ONFH-BMMSC group. (A) Normal group. (B) ONFH-BMMSC group, (C) LiCl + ONFH-BMMSC group. (D) OD values of Alizarin Red S staining in the $\mathrm{LiCl}+\mathrm{ONFH}-\mathrm{BMMSC}$ group and the normal group were significantly higher than those of the ONFH-BMMSC group. The data are shown as the means $\pm \mathrm{SD}$. ${ }^{* * *} \mathrm{P}<0.001$ compared with the ONFH-BMMSC group. $\mathrm{LiCl}$, lithium chloride.

found that the OCN and Runx2 mRNA levels did not significantly differ between the $\mathrm{LiCl}+\mathrm{ONFH}-\mathrm{BMMSC}$ group and the normal BMMSC group ( $\mathrm{P}>0.05$; Fig. 2).

The results revealed that the normal and the $\mathrm{LiCl}+\mathrm{ONFH}-$ BMMSC groups presented with a greater number of mineralized nodules than the ONFH-BMMSC group (Fig. 3A-C). Furthermore, the light absorbance analysis revealed that the normal and $\mathrm{LiCl}+\mathrm{ONFH}-\mathrm{BMMSC}$ groups had significantly higher OD values than the ONFH-BMMSC group $(\mathrm{P}<0.001)$; by contrast, the OD values did not differ between the normal and the $\mathrm{LiCl}+\mathrm{ONFH}-\mathrm{BMMSC}$ groups $(\mathrm{P}>0.05$; Fig. 3D).

$\mathrm{LiCl}$ reduces the adipogenesis of ONFH-BMMSCs. The $\mathrm{LiCl}$ treatment group and normal group exhibited a significant decrease in the mRNA expression of PPAR $\gamma$ and Fabp4 compared with the ONFH-BMMSC group $(\mathrm{P}<0.001$, Fig. 4). 

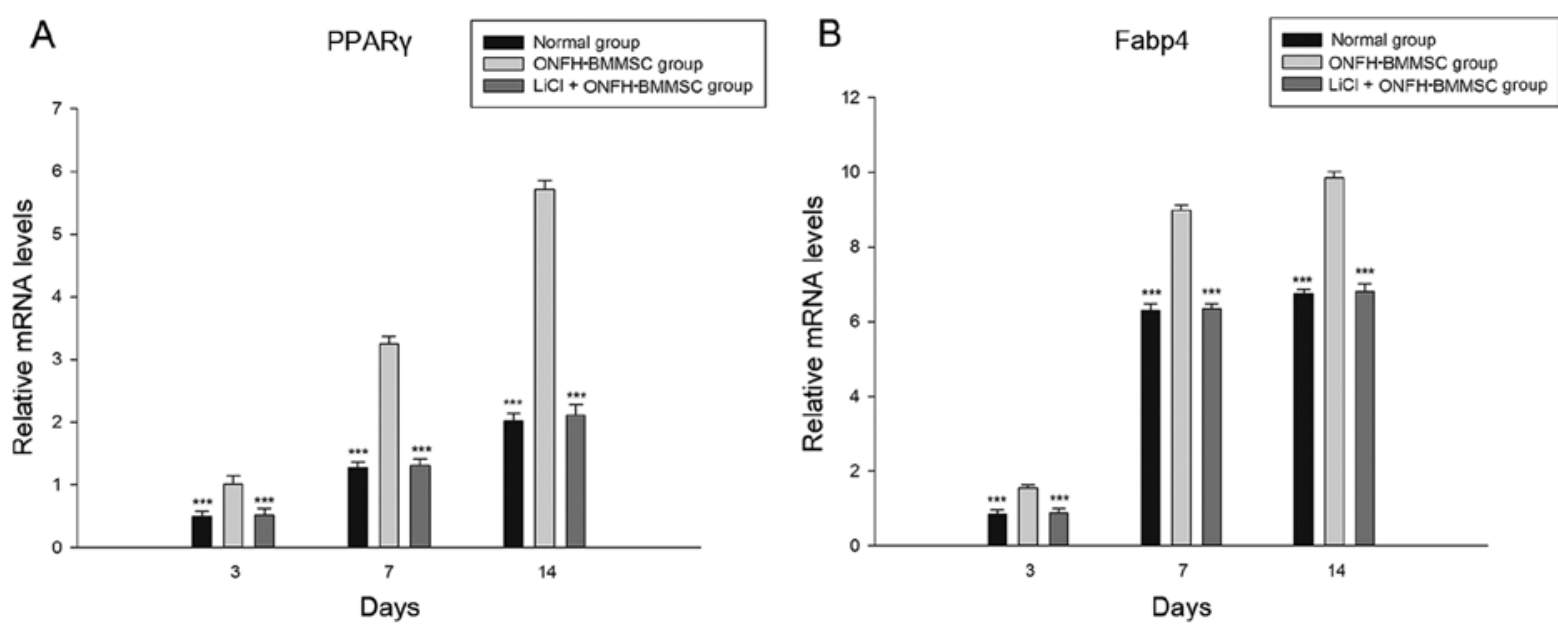

Figure 4. RT-qPCR of adipogenesis-related markers. RT-qPCR was used to determine the adipogenic effects of bone marrow-derived mesenchymal stem cells (BMMSCs) on the mRNA expression of (A) peroxisome proliferator-activated receptor $\gamma$ (PPAR $\gamma$ ) and (B) fatty acid binding protein 4 (Fabp4) at different time points during adipogenic induction. Gene expression was normalized to $\beta$-actin. The data are shown as the means \pm SD, ${ }^{* * * *} \mathrm{P}<0.001$ compared with the BMMSCs isolated from rats with steroid-related osteonecrosis of the femoral head (ONFH-BMMSC) group. LiCl, lithium chloride.
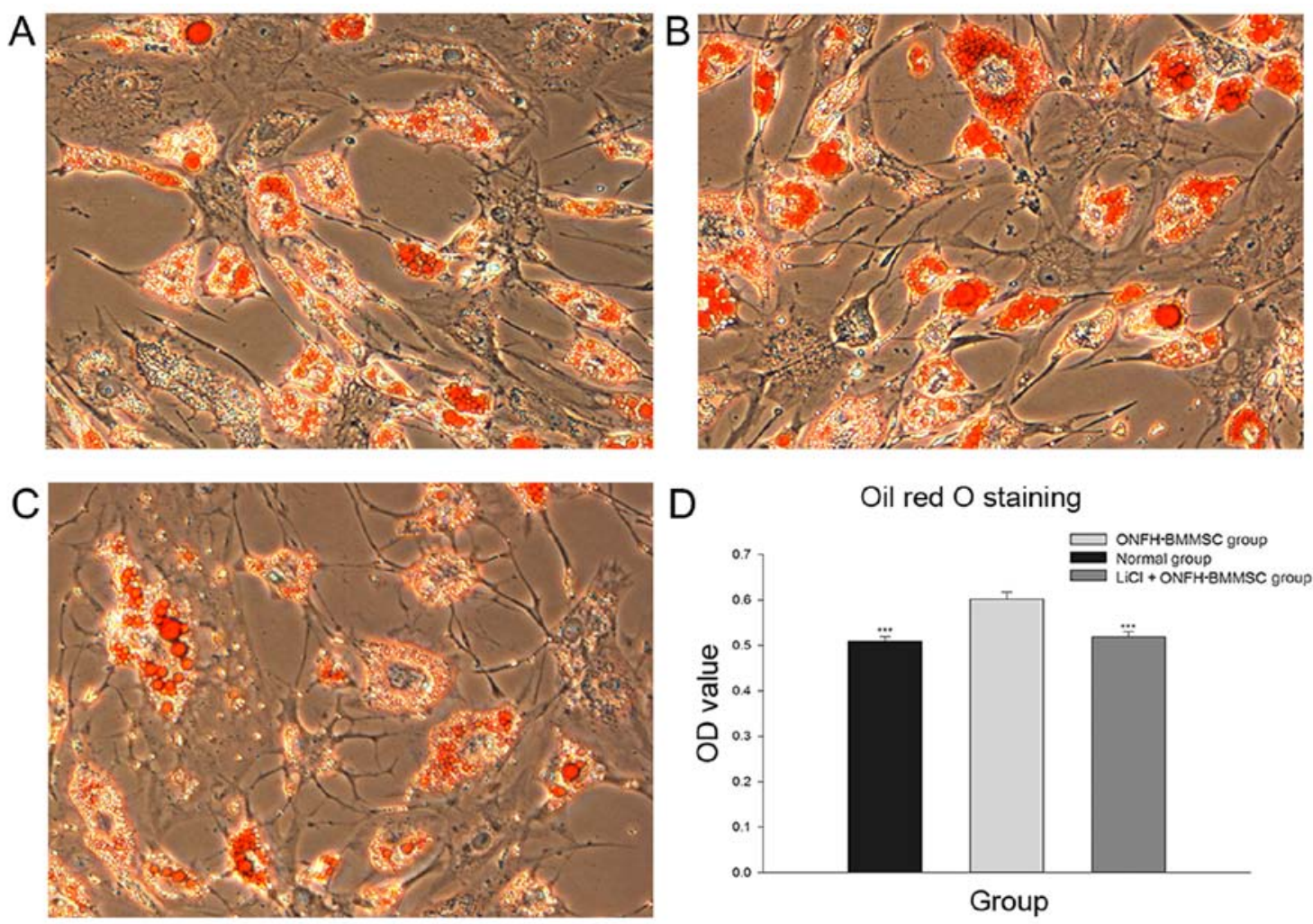

Figure 5. Oil Red O staining for lipid droplet formation. The bone marrow-derived mesenchymal stem cells isolated from rats with steroid-related osteonecrosis of the femoral head (ONFH-BMMSC) group showed heavier Oil Red O staining when compared with the normal group or the LiCl + ONFH-BMMSC group. (A) Normal group. (B) ONFH-BMMSC group, (C) $\mathrm{LiCl}+$ ONFH-BMMSC group. (D) OD values of Oil Red O staining in the $\mathrm{LiCl}+\mathrm{ONFH}-\mathrm{BMMSC}$ group and the normal group were significantly lower than the ONFH-BMMSC group. The data are shown as the means \pm SD. ${ }^{* * *} \mathrm{P}<0.001$ compared with the ONFHBMMSC group. LiCl, lithium chloride.

Furthermore, we found that the $\mathrm{LiCl}$ treatment group showed no statistically significant differences in the PPAR $\gamma$ and Fabp4 mRNA expression levels compared with the normal BMMSC group ( $\mathrm{P}>0.05$; Fig. 4).

The results obtained from Oil Red O staining revealed that the normal and $\mathrm{LiCl}+\mathrm{ONFH}-\mathrm{BMMSC}$ groups presented with fewer lipid droplets than the ONFH-BMMSC group (Fig.5A-C). Moreover, the light absorbance analysis revealed that the normal and $\mathrm{LiCl}+\mathrm{ONFH}-\mathrm{BMMSC}$ groups had significantly lower OD values than the ONFH-BMMSC group $(\mathrm{P}<0.001)$, while the $\mathrm{OD}$ values between the normal and the $\mathrm{LiCl}+\mathrm{ONFH}-$ BMMSC groups did not differ ( $\mathrm{P}>0.05$; Fig. 5D).

LiCl activates the $\beta$-catenin pathway. As shown in Fig. 6, treatment with $\mathrm{LiCl}$ markedly increased the expression of $\beta$-catenin and decreased the p-Tyr216 GSK-3 $\beta$ protein levels compared 
A
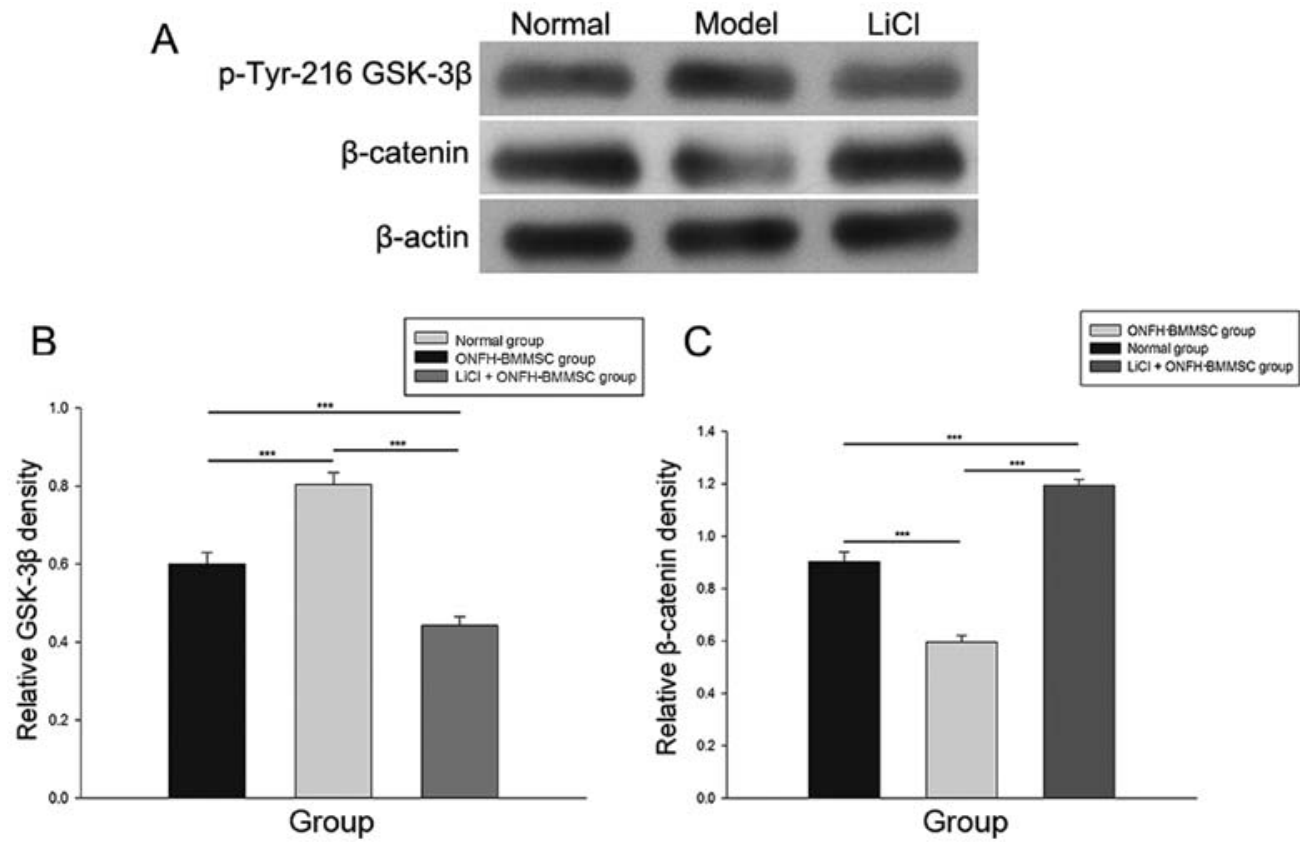

Figure 6. Western blot analysis of the $\beta$-catenin pathway. (A) Protein expression levels of p-Tyr216 GSK-3 $\beta$ and $\beta$-catenin were measured by western blot analysis. The bar graphs show the relative expression of (B) $\mathrm{p}$-Tyr216 GSK-3 $\beta$ and (C) $\beta$-catenin. The data are represented as the means $\pm \mathrm{SD},{ }^{* * *} \mathrm{P}<0.001$. LiCl, lithium chloride; ONFH-BMMSC group, group of bone marrow-derived mesenchymal stem cells isolated from rats with steroid-related osteonecrosis of the femoral head.
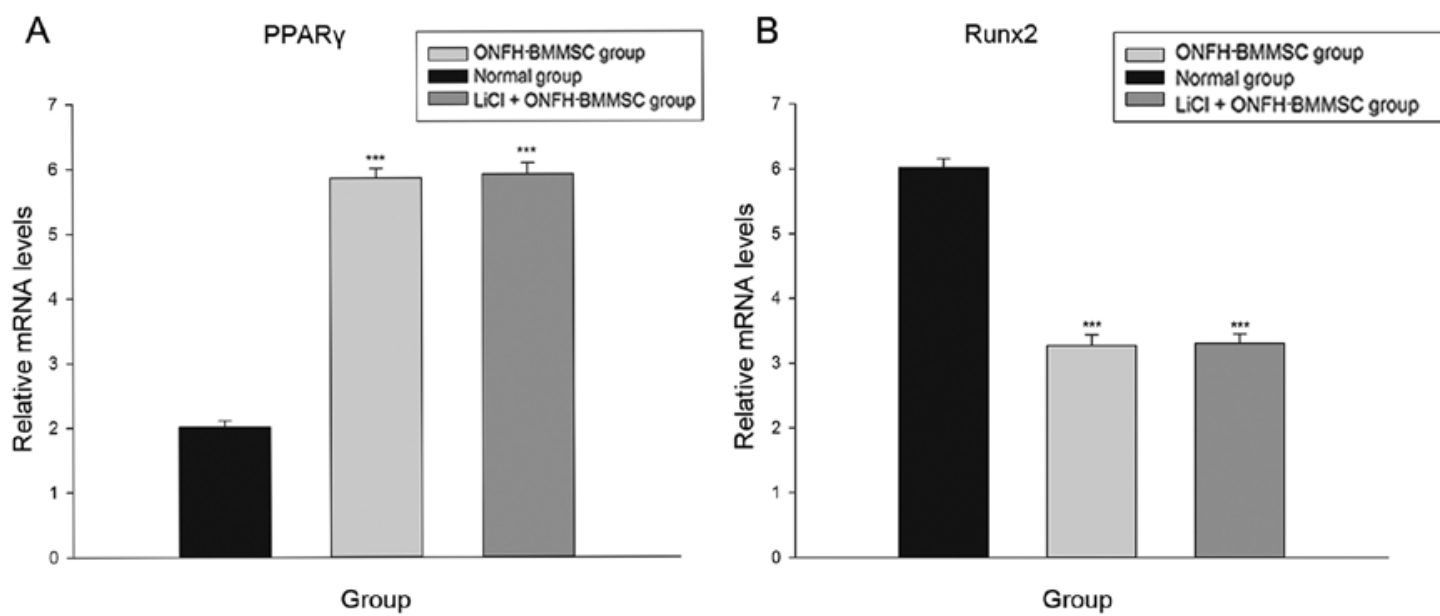

Figure 7. RT-qPCR of adipogenic/osteogenic markers. RT-qPCR was used to determine the adipogenic and osteogenic effects of different treatments for bone marrow-derived mesenchymal stem cells (BMMSCs), isolated from rats with steroid-related osteonecrosis of the femoral head (ONFH), on the mRNA expression of (A) peroxisome proliferator-activated receptor $\gamma$ PPAR $\gamma$ and (B) Runx2 at the end of adipogenic and osteogenic induction. Gene expression was normalized to $\beta$-actin. The data are shown as the means $\pm \mathrm{SD},{ }^{* * *} \mathrm{P}<0.001$ compared with the $\mathrm{LiCl}+\mathrm{ONFH}-\mathrm{BMMSC}$ group. LiCl, lithium chloride; Que, quercetin.

with the ONFH-BMMSC group $(\mathrm{P}<0.001)$. The normal group exhibited a higher expression of $\beta$-catenin and a lower expression of $\mathrm{p}$-Tyr216 GSK-3 $\beta$ compared with the ONFH-BMMSC group $(\mathrm{P}<0.001)$.

Inhibition of $\beta$-catenin pathway diminishes the effects of $\mathrm{LiCl}$. The $\mathrm{LiCl}+\mathrm{Que}+\mathrm{ONFH}-\mathrm{BMMSC}$ and ONFH-BMMSC groups exhibited a significant increase in the mRNA expression of PPAR $\gamma$ compared with the $\mathrm{LiCl}+\mathrm{ONFH}$ group $(\mathrm{P}<0.001$; Fig. 7A). By contrast, the $\mathrm{LiCl}+$ Que + ONFH-BMMSC and ONFH-BMMSC groups exhibited a statistically significant decrease in the mRNA expression of Runx $2(\mathrm{P}<0.001$; Fig. 7B). Moreover, the $\mathrm{LiCl}+$ Que + ONFH-BMMSC group exhibited no statistically significant difference in PPAR $\gamma$ and Runx2 mRNA levels compared with the ONFH-BMMSC group ( $\mathrm{P}>0.05$; Fig. 7 ).

Immunofluorescence staining revealed that the $\mathrm{LiCl}+\mathrm{Que}+\mathrm{ONFH}-\mathrm{BMMSC}$ and ONFH-BMMSC groups exhibited a significant increase in Fabp4 expression compared with the $\mathrm{LiCl}+\mathrm{ONFH}$ group $(\mathrm{P}<0.001$; Fig. 8A) By contrast, the $\mathrm{LiCl}+$ Que + ONFH-BMMSC and ONFHBMMSC groups exhibited a significantly lower expression of OCN (P<0.001; Fig. 8B). Moreover, we also found that the $\mathrm{LiCl}+\mathrm{Que}+\mathrm{ONFH}-\mathrm{BMMSC}$ group exhibited no statistically significant difference in Fabp4 and OCN expression compared with the ONFH-BMMSC group ( $\mathrm{P}>0.05$; Fig. 8 ). 


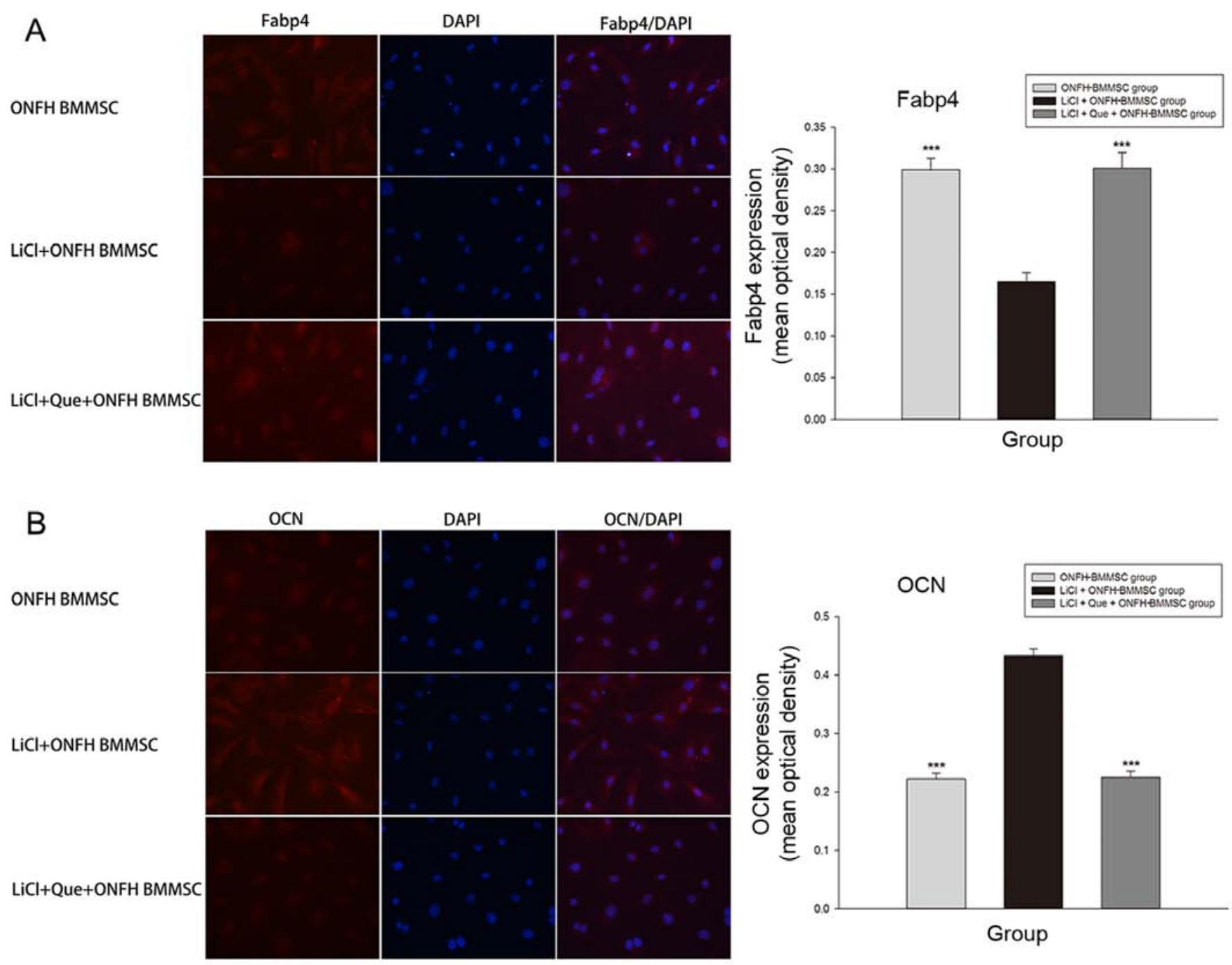

Figure 8. Immunofluorescence staining for adipogenic/osteogenic markers. (A and B) Immunofluorescence staining for fatty acid binding protein 4 (Fabp4) and osteocalcin (OCN) (TRITC-Red) with DAPI (blue) nuclear counterstaining (DAPI, blue) at the end of adipogenic/osteogenic induction (magnification, x400). The data are shown as the means $\pm \mathrm{SD},{ }^{* * *} \mathrm{P}<0.001$ compared with the $\mathrm{LiCl}+\mathrm{ONFH}-\mathrm{BMMSC}$ group; BMMSCs, bone marrow-derived mesenchymal stem cells isolated from rats with steroid-related osteonecrosis of the femoral head; $\mathrm{LiCl}$, lithium chloride.

\section{Discussion}

ONFH is a common degenerative disease that mostly occurs in young and middle age. The disease critically affects the quality of life of patients and is associated with a high morbidity. Hernigou et al demonstrated that the decreased activity of BMMSCs may contibute to the pathogenesis of ONFH (4). In a prevoius study, in patients who developed osteonecrosis following treatment with corticosteroids, abnormal adipogenesis was discovered in the bone marrow, with a decreased number of osteogenic cells (21). These findings indicate that steroids may disrupt the osteogenic/adipogenic activity of stem cells in ONFH.

To explore the potential impairment in the osteogenic/adipogenic activity of BMMSCs in ONFH, we established a rat model of steroid-related ONFH previously reported by Chen et al (17). According to their study, the necrosis generated in this model is similar to that observed in steroid-treated patients. In the present study, Alizarin Red S staining determined the osteogenic function of BMMSCs in the model group, which was impaired compared with the normal BMMSCs. By contrast, Oil Red O staining indicated that the adipogenic function of BMMSCs isolated from rats with steroid-related ONFH was enhanced compared with normal BMMSCs. These results indicated that the differentiation of ONFH-BMMSCs was impaired, which may be the cause of steroid-related ONFH.

Lithium has been used in the treatment of bipolar disorder for decades, and its safety has been well confirmed (22). Studies have reported that lithium inhibits the adipogenic effect $(15,16)$. Mai et al also confirmed the anti-adipogenic effects of $\mathrm{LiCl}$ (23). $\mathrm{LiCl}$ has also been reported to stimulate osteoblast differentiation (14). However, it remains unknown as to whether lithium can reverse the abnormal osteogenic/ adipogenic activity of BMMSCs from rats with steroid-related ONFH. In the present study, we examined the possibility of using $\mathrm{LiCl}$ to reverse the abnormal differentiation of ONFHBMMSCs. The effects of lithium have been reported to be highly dependent on its concentration $(24,25)$; thus, we tested a series of $\mathrm{LiCl}$ concentrations to find the optimal concentration. We found that $5 \mathrm{mM} \mathrm{LiCl}$ exerted the greatest stimulatory 
effect on the viability of ONFH-BMMSCs. Thus, we selected $5 \mathrm{mM} \mathrm{LiCl}$ as the optimal concentration.

Runx 2 is essential for osteoblast differentiation and skeletal morphogenesis. Moreover, OCN, also known as bone gamma-carboxyglutamic acid-containing protein (BGLAP), is a non-collagenous protein found in bone (26). It has been implicated in bone mineralization and calcium ion homeostasis (27). In the present study, we found the decreased expression of Runx 2 and OCN in the ONFH-BMMSCs, suggesting that steroid-related ONFH reduces the osteogenic activity of BMMSCs. Following treatment with $\mathrm{LiCl}$, the expression of Runx 2 and OCN significantly increased, and this was consistent with the results of Alizarin Red S staining. These results indicate that $\mathrm{LiCl}$ can reverse the abnormal osteogenic differentiation of ONFH-BMMSCs.

By contrast, PPAR $\gamma$, as a member of the nuclear receptor gene superfamily of ligand-activated transcription factors, functions as a stimulator of bone marrow adipogenesis and an inhibitor of osteogenesis. Fabp4 is a carrier protein for fatty acids that is considered to be a terminal differentiation marker for adipocytes (28). In the present study, we found the increased expression of PPAR $\gamma$ and Fabp4 in the BMMSCs from rats with steroid-related ONFH, indicating that steroid-related ONFH may cause BMMSCs to change into an adipogenic phenotype. Following treatment with $\mathrm{LiCl}$, the expression of PPAR $\gamma$ and Fabp4 significantly decreased. These results were consistent with those of Oil Red O staining, suggesting that lithium attenuates the impairment of excessive adipogenic differentiation of ONFH-BMMSCs.

Lithium, as used in the present study, is known to be a GSK-3 $\beta$ inhibitor that can raise the $\beta$-catenin concentration (14). The $\mathrm{Wnt} / \beta$-catenin pathway has been reported to inhibit adipocyte differentiation (29), as well as enhance osteoblast differentiation (30). As a key component of this pathway, the activation of $\beta$-catenin leads to the direct suppression of PPAR $\gamma$ and the prevention of 3T3-L1 cell adipogenic differentiation $(31,32)$. By contrast, the disruption of the $\beta$-catenin pathway increases adipogenesis (33) and reduces bone formation (29). In addition, GSK-3 $\beta$ activity is regulated by phosphorylation and Tyr216 phosphorylation increases its activity (34) and Garza et al (35) reported that steroids inhibit GSK-3 $\beta / \beta$-catenin signaling by activating GSK-3 $\beta$. This activation inhibited the activity of $\beta$-catenin (35) and was consistent with our present findings that the ONFH-BMMSCs had decreased levels of $\beta$-catenin and increased levels of p-Tyr216 GSK-3 $\beta$ (active form of GSK-3 $\beta$ ) compared with the normal BMMSCs. The results of western blot analysis in the present study revealed that $\mathrm{LiCl}$, as a GSK-3 $\beta$ inhibitor, activated $\beta$-catenin by reducing $\mathrm{p}$-Tyr 216 GSK-3 $\beta$. However, whether lithium attenuated the abnormal osteogenic/adipogenic differentiation of ONFH-BMMSCs through the $\beta$-catenin pathway remains uncertain. To further elucidate the possible mechanisms of action of lithium in ONFH-BMMSCs, we used Que, an inhibitor of the $\beta$-catenin pathway. We found that the treatment effects of $\mathrm{LiCl}$ (the attenuation of the abnormal osteogenic and adipogenic differentiation) on ONFH-BMMSCs were abolished by treatment with Que. In this respect, we speculated that lithium may act as a switch to reduce both the abnormal adipogenic activity and increase the osteogenic differentiation of ONFH-BMMSCs by activating the $\beta$-catenin pathway.
In conclusion, our data indicate that the normal osteogenic/ adipogenic activity of BMMSCs is impaired during steroidrelated ONFH. The present study also provides evidence to suggest that $\mathrm{LiCl}$ attenuates the abnormal osteogenic/adipogenic differentiation of BMMSCs obtained from rats with steroidrelated ONFH by activating the $\beta$-catenin signaling pathway. These findings highlight the treatment effects of $\mathrm{LiCl}$ on steroidrelated ONFH and provide an effective therapeutic strategy.

\section{Acknowledgements}

The present study was supported by the National Natural Science Foundation of China (grant nos. 81101363, 81371944 and 81572145) and the Fundamental Research Funds for the Central Universities.

\section{References}

1. Sun Z, Yang S, Ye S, Zhang Y, Xu W, Zhang B, Liu X, Mo F and Hua W: Aberrant CpG islands' hypermethylation of ABCB1 in mesenchymal stem cells of patients with steroid-associated osteonecrosis. J Rheumatol 40: 1913-1920, 2013.

2. Lieberman JR, Berry DJ, Mont MA, Aaron RK, Callaghan JJ, Rajadhyaksha AD and Urbaniak JR: Osteonecrosis of the hip: management in the 21st century. Instr Course Lect 52: 337-355, 2003.

3. Webster RA, Blaber SP, Herbert BR, Wilkins MR and Vesey G: The role of mesenchymal stem cells in veterinary therapeutics - a review. N Z Vet J 60: 265-272, 2012.

4. Hernigou P and Beaujean F: Abnormalities in the bone marrow of the iliac crest in patients who have osteonecrosis secondary to corticosteroid therapy or alcohol abuse. J Bone Joint Surg Am 79: 1047-1053, 1997.

5. Seamon J, Keller T, Saleh J and Cui Q: The pathogenesis of nontraumatic osteonecrosis. Arthritis (Egypt) 2012: 601763, 2012.

6. Abdallah BM and Kassem M: New factors controlling the balance between osteoblastogenesis and adipogenesis. Bone 50: 540-545, 2012.

7. Sheng H, Sheng CJ, Cheng XY, Zhang G, Lee KM, Leung KS, $\mathrm{Qu} \mathrm{S}$ and Qin L: Pathomorphological changes of bone marrow adipocytes in process of steroid-associated osteonecrosis. Int $\mathrm{J}$ Clin Exp Pathol 6: 1046-1050, 2013.

8. Taipaleenmäki H, Abdallah BM, AlDahmash A, Säämänen AM and Kassem M: Wnt signalling mediates the cross-talk between bone marrow derived pre-adipocytic and pre-osteoblastic cell populations. Exp Cell Res 317: 745-756, 2011.

9. Franceschi RT and Xiao G: Regulation of the osteoblast-specific transcription factor, Runx2: responsiveness to multiple signal transduction pathways. J Cell Biochem 88: 446-454, 2003.

10. Liu C, Li Y, Semenov M, Han C, Baeg GH, Tan Y, Zhang Z, Lin $\mathrm{X}$ and He X: Control of beta-catenin phosphorylation/degradation by a dual-kinase mechanism. Cell 108: 837-847, 2002.

11. Clevers $H$ and Nusse $R$ : Wnt $/ \beta$-catenin signaling and disease. Cell 149: 1192-1205, 2012.

12. Young W: Review of lithium effects on brain and blood. Cell Transplant 18: 951-975, 2009.

13. Wexler EM, Geschwind DH and Palmer TD: Lithium regulates adult hippocampal progenitor development through canonical Wnt pathway activation. Mol Psychiatry 13: 285-292, 2008.

14. Galli C, Piemontese M, Lumetti S, Manfredi E, Macaluso GM and Passeri G: GSK3b-inhibitor lithium chloride enhances activation of Wnt canonical signaling and osteoblast differentiation on hydrophilic titanium surfaces. Clin Oral Implants Res 24: 921-927, 2013.

15. Itoigawa $\mathrm{Y}, \mathrm{Kishimoto} \mathrm{KN}$, Sano $\mathrm{H}$, Kaneko $\mathrm{K}$ and Itoi $\mathrm{E}$ : Molecular mechanism of fatty degeneration in rotator cuff muscle with tendon rupture. J Orthop Res 29: 861-866, 2011.

16. Sen B, Xie Z, Case N, Ma M, Rubin C and Rubin J: Mechanical strain inhibits adipogenesis in mesenchymal stem cells by stimulating a durable beta-catenin signal. Endocrinology 149: 6065-6075, 2008.

17. Chen S, Li J, Peng H, Zhou J and Fang H: Administration of erythropoietin exerts protective effects against glucocorticoidinduced osteonecrosis of the femoral head in rats. Int J Mol Med 33: 840-848, 2014. 
18. Panepucci RA, Siufi JLC, Silva WA Jr, Proto-Siquiera R, Neder L, Orellana M, Rocha V, Covas DT and Zago MA: Comparison of gene expression of umbilical cord vein and bone marrow-derived mesenchymal stem cells. Stem Cells 22: 1263-1278, 2004.

19. Boku S, Nakagawa S, Masuda T, Nishikawa H, Kato A, Kitaichi Y, Inoue $\mathrm{T}$ and Koyama T: Glucocorticoids and lithium reciprocally regulate the proliferation of adult dentate gyrus-derived neural precursor cells through GSK-3beta and beta-catenin/TCF pathway. Neuropsychopharmacology 34: 805-815, 2009.

20. Park CH, Chang JY, Hahm ER, Park S, Kim HK and Yang CH: Quercetin, a potent inhibitor against beta-catenin/Tcf signaling in SW480 colon cancer cells. Biochem Biophys Res Commun 328 227-234, 2005.

21. Cui Q, Wang GJ and Balian G: Pluripotential marrow cells produce adipocytes when transplanted into steroid-treated mice. Connect Tissue Res 41: 45-56, 2000.

22. Jope RS: Lithium and GSK-3: One inhibitor, two inhibitory actions, multiple outcomes. Trends Pharmacol Sci 24: 441-443, 2003.

23. Mai Y, Zhang Z, Yang H, Dong P, Chu G, Yang G and Sun S: BMP and activin membrane-bound inhibitor (BAMBI) inhibits the adipogenesis of porcine preadipocytes through Wnt/beta-catenin signaling pathway. Biochem Cell Biol 92: 172-182, 2014.

24. De Boer J, Wang HJ and Van Blitterswijk C: Effects of Wnt signaling on proliferation and differentiation of human mesenchymal stem cells. Tissue Eng 10: 393-401, 2004.

25. Gregory CA, Perry AS, Reyes E, Conley A, Gunn WG and Prockop DJ: Dkk-1-derived synthetic peptides and lithium chloride for the control and recovery of adult stem cells from bone marrow. J Biol Chem 280: 2309-2323, 2005.

26. Makita N, Suzuki M, Asami S, Takahata R, Kohzaki D, Kobayashi S, Hakamazuka T and Hozumi N: Two of four alternatively spliced isoforms of RUNX2 control osteocalcin gene expression in human osteoblast cells. Gene 413: 8-17, 2008.

27. Lin GT, Tseng HF, Chang CK, Chuang LY, Liu CS, Yang CH, Tu CJ, Wang EC, Tan HF, Chang CC, et al: SNP combinations in chromosome-wide genes are associated with bone mineral density in Taiwanese women. Chin J Physiol 51: 32-41, 2008.
28. Mackay DL, Tesar PJ, Liang LN and Haynesworth SE: Characterizing medullary and human mesenchymal stem cell-derived adipocytes. J Cell Physiol 207: 722-728, 2006.

29. Wang XL, Wang N, Zheng LZ, Xie XH, Yao D, Liu MY, Yao ZH, Dai Y, Zhang G, Yao XS and Qin L: Phytoestrogenic molecule desmethylicaritin suppressed adipogenesis via Wnt/ $\beta$-catenin 1signaling pathway. Eur J Pharmacol 714: 254-260, 2013.

30. Corrado A, Neve A, Macchiarola A, Gaudio A, Marucci A and Cantatore FP: RANKL/OPG ratio and DKK-1 expression in primary osteoblastic cultures from osteoarthritic and osteoporotic subjects. J Rheumatol 40: 684-694, 2013.

31. Ross SE, Hemati N, Longo KA, Bennett CN, Lucas PC, Erickson RL and MacDougald OA: Inhibition of adipogenesis by Wnt signaling. Science 289: 950-953, 2000.

32. Liu J and Farmer SR: Regulating the balance between peroxisome proliferator-activated receptor gamma and beta-catenin signaling during adipogenesis. A glycogen synthase kinase 3beta phosphorylation-defective mutant of beta-catenin inhibits expression of a subset of adipogenic genes. J Biol Chem 279: 45020-45027, 2004.

33. Bennett CN, Ross SE, Longo KA, Bajnok L, Hemati N, Johnson KW, Harrison SD and MacDougald OA: Regulation of Wnt signaling during adipogenesis. J Biol Chem 277: 30998-31004, 2002.

34. Hughes K, Nikolakaki E, Plyte SE, Totty NF and Woodgett JR: Modulation of the glycogen synthase kinase- 3 family by tyrosine phosphorylation. EMBO J 12: 803-808, 1993.

35. Garza JC, Guo M, Zhang W and Lu XY: Leptin restores adult hippocampal neurogenesis in a chronic unpredictable stress model of depression and reverses glucocorticoid-induced inhibition of GSK-3 $\beta / \beta$-catenin signaling. Mol Psychiatry 17: 790-808, 2012. 